\title{
Joint moment loading interplay between the shoulders and the low back during patient handling in nurses
}

\author{
Alicia L. Nadon, Alan C. Cudlip and Clark R. Dickerson* \\ Department of Kinesiology, University of Waterloo, Waterloo, ON, Canada
}

\begin{abstract}
.
BACKGROUND: Manual patient handling completed by nurses can include unexpected or strenuous exertions, potentially leading to injury. Lifting guidelines focus on mitigating primarily limiting low back exposures, and the influence of these techniques on the shoulder is unknown.

OBJECTIVE: To quantify shoulder loading during manual patient handling tasks and determine whether approaches intended to limit low back exposures negatively affect shoulder demands.

METHODS: Twenty novice university-aged females completed five manual patient handling tasks before and after a training session. Participants simulated handling a partial weight bearing patient, and joint load magnitudes were calculated. Strength demands were interpreted in the context of available population capability ranges.

RESULTS: Using recommended techniques decreased peak low back loading in most scenarios $(p=0.01-0.02)$, but had variable effects on shoulder loading. Peak loading in the shoulders increased by $97-107 \%$ in the Sit-to-Chair task following training. Using recommended techniques for Turn Toward decreased mean population strength requirements at the back to $35 \%$ of capability while increasing shoulder requirements to $100 \%$ capability.

CONCLUSIONS: Recommended manual patient handling techniques mitigate low back exposures but likely transfer demands to other body regions, specifically the shoulder.
\end{abstract}

Keywords: Nursing, manual patient handling, upper extremity, joint loading

\section{Introduction}

Manual materials handling is often associated with manufacturing and warehousing, but nontraditional load handling scenarios exist in other workplaces, and are accompanied by the same musculoskeletal overexertion potential. Indeed, the manual patient handling completed by nurses requires transferring or repositioning substantially more complicated, unmodifiable, and often heavier objects.

Differences in anthropometrics, ability to assist, or combative and uncooperative patients can result in unexpected or strenuous exertions, leading to injury risk [1-3]. Health care and social services are second only to manufacturing sectors in Ontario for lost time injury claims [4], and musculoskeletal disorders are the cause for $\sim 50 \%$ of injuries in this group, nine times the US national rate [5].

\footnotetext{
${ }^{*}$ Corresponding author: Clark Dickerson, Department of Kinesiology, University of Waterloo, 200 University Ave. W, Waterloo, ON N2N 2T8, Canada. Tel.: +1 5198884567 x37844; Fax: +1 519746 6776; E-mail: cdickers@uwaterloo.ca.
} 
Injury rates led to extensive research and creation of standardized lifting practices, but adoption of these practices can be slow, and practices focus almost exclusively on the low back. Specific tasks, including turning, repositioning or transferring a patient are recognized as high-demand activities, producing greater than recommended spinal compression forces [6-9]. The dangers associated with manual patient handling have led many health units to adopt safe lifting policies, and in some cases banning certain patient handling tasks [3]. Mechanical lifting devices and lifting techniques have effectively reduced low back injuries and lost days from work $[2,9,10]$. Despite these advances, manual patient handling continues to be completed by nurses who cite a lack of time, unfamiliarity with the manual devices and a lack of available equipment as reasons for favouring manual transfers [11-13]. Widespread adoption of these techniques can be slow; previous research has indicated that up to $46 \%$ of registered nurses either were not informed or aware of national safe patient handling and mobility standards [14]. While nurses receive training throughout their undergraduate careers as well as on the job training, they often mimic the lifting techniques performed by more experienced persons $[15,16]$; which may result from the new nurse wanting to assimilate and avoid confrontation. Thus, new nurses may continue to use suboptimal techniques and promote these strategies amongst subsequent nursing generations [17,18].

While developed strategies have reduced low back injury in nurses, the influence of these techniques on the shoulder is unknown. As these ergonomic interventions have focused chiefly on the low back, concerns exist regarding potential physical demand transfers between joint in manual patient handling [19-21]. Some tasks, such as shifting the patient up or down the bed or turning the patient to one side, are highly demanding of the shoulder [20]. Other intended ergonomics interventions reduced perceived exertion at the low back while increasing it at the shoulder [2,10]. Shoulder injuries in manual patient handling remain common among nurses. The focus of this work is to quantify shoulder loading during manual patient handling tasks and determine whether approaches intended to reduce low back exposures concomitantly increase shoulder demands. We hypothesized that trained manual patient handling techniques would decrease low back loading, but would increase peak joint moments observed at the shoulders.

\section{Methods}

\subsection{Participants}

Twenty healthy university-aged females $(21.6 \pm 1.3$ years; $1.66 \pm 0.07 \mathrm{~m}$ tall; $62.5 \pm 9.4 \mathrm{~kg}$ body weight) participated. Exclusion criteria were injury or pain in the past year to the upper extremity or back and previous experience or training in manual patient handling. Participants provided informed consent prior to collection, and the institutional Office of Research Ethics approved the study.

\subsection{Instrumentation}

Motion capture data was collected for all experimental trials. Three-dimensional motion was tracked using an 8-camera Vicon MX20 system (VICON, Oxford, UK). Individual markers were placed over anatomical landmarks: bilaterally over the $2^{\text {nd }}$ and $5^{\text {th }}$ metacarpals, radial and ulnar styloids, medial and lateral epicondyles, acromii, and posterior superior iliac spine, as well as on the suprasternal notch, xiphoid process, and $5^{\text {th }}$ lumbar spinous process. A static calibration frame established relationships between clusters and anatomical landmark markers, allowing subsequent construction of segment coordinate systems and joint centers [22]. Kinematics were sampled at $50 \mathrm{~Hz}$ using VICON Nexus 1.2.1 software (Oxford, UK). 


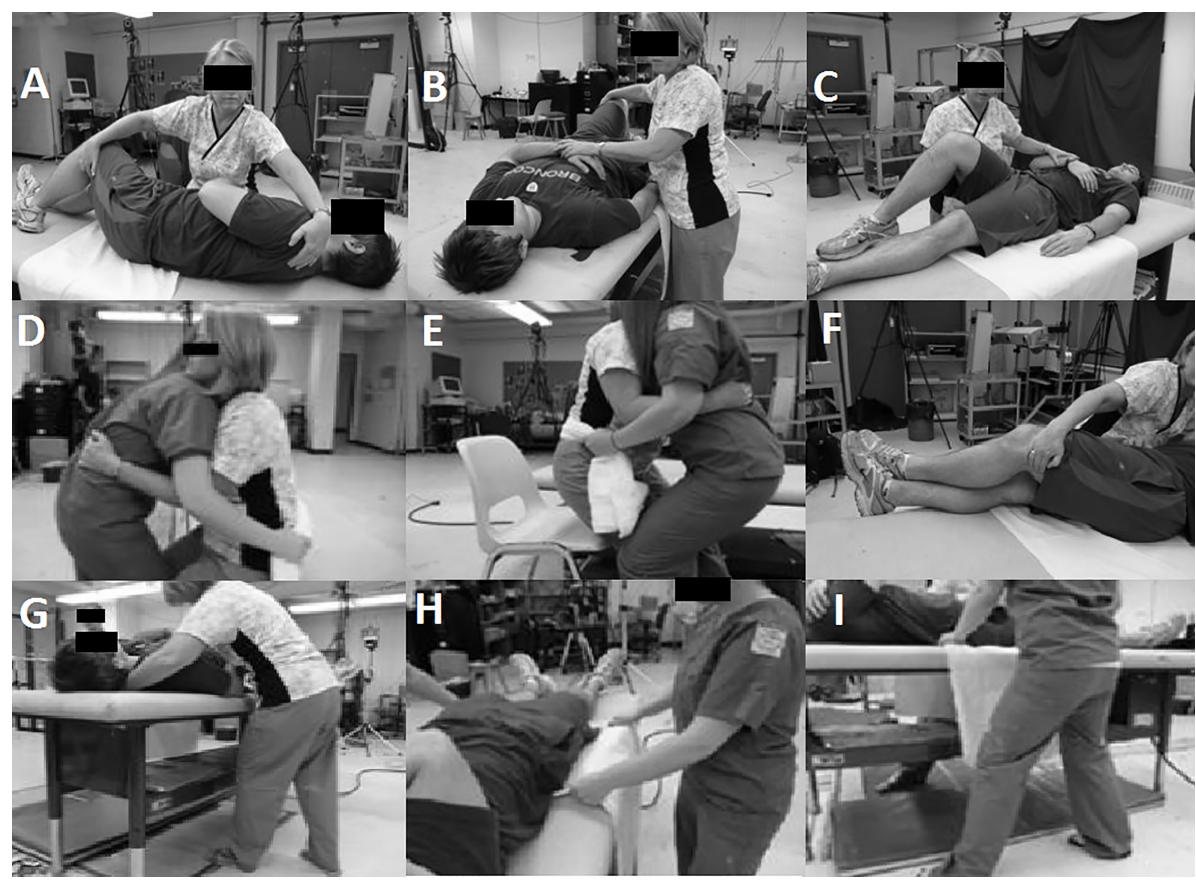

Fig. 1. Example manual patient handling pictures that would accompany detailed descriptions on how to properly perform the recommended techniques. A: Turn Toward initiation; B: Turn Toward; C: Turn Away; D: Sit-to-Chair lift; E: Sit-to-Chair lower; F: Lie-to-Sit hand position; G: Lie-to-Sit foot position; H: Reposition hand position; I: Reposition foot position.

To quantify resultant joint moments at the shoulders and low back, forces generated at the hand were estimated following each set of manual patient handling trials. Two ErgoFet $300^{\mathrm{TM}}$ uniaxial hand dynamometers (Hogan Health Industries, Utah, USA) were used to record hand force estimates for each task. Before collection, participants were calibrated with the dynamometers to determine left and right hand maximum pushes and pulls in similar postures as they assumed in experimental trials to improve accuracy [23]. This calibration involved familiarization of the exertion levels required to attain $25 \%$, $50 \%$ and $75 \%$ of their maximal force outputs.

\subsection{Experimental design}

Participants performed defined manual patient handling tasks in two sessions on the same day, before and after a training session. The training session included a self-paced computer presentation of recommended techniques (described graphically and verbally) and videos of recommended lifting strategies for each task. Each video included a practicing nurse with 15 years of experience demonstrating the recommended techniques. The training session finished with physical familiarization and practice with the techniques, including manipulating the patient and feedback from the instructor. Task-specific recommended techniques were extracted from the literature [9,24] (Fig. 1).

\subsection{Protocol}

The protocol involved application of reflective markers and hand force calibration, a pre-training session, training of recommended techniques, then a post-training session. Within each session, five tasks 
were block-randomized and performed three times each for a total of 30 trials ( 15 pre- and post-training), with rest breaks of up to 5 minutes between tasks. The five tasks analysed were: turning the patient toward and away from the nurse (Turn Toward and Turn Away, respectively), moving the patient upward on the bed (Reposition), moving the patient from supine to sitting on the edge of the bed (Lie-to-Sit), and transferring the patient from sitting on the bed to sitting on an adjacent chair (Sit-to-Chair).

Task conditions were consistent across participants. Each trial was completed on an electrically adjustable cushioned table (56-88 $\mathrm{cm}$ above the floor) covered in a slider sheet similar to those found in hospitals. Table height was placed at any height the participant chose during untrained trials, while participants used their waist height in post-training trials, except for the Sit-to-Chair task when the height of the table was moved to match the height of the seat pan of the chair. A 92-kg male was used as the "patient" throughout data collection, and was $87^{\text {th }}$ percentile based on anthropometry tables for weight, and $72^{\text {nd }}$ percentile for stature [25]. The patient simulated a partial weight bearing patient, and only supported himself when in a seated or standing position. The participant always approached the bed on the left, with the head of the patient to the left and feet to the right. Before the pre-training session, the participant was given time to decide on their self-selected technique for moving the patient, along with instruction on the starting and ending patient positions for each task and the opportunity to attempt a few transfers. Specific instructions following training were emphasized during the post-training trials, including guidance to stay as close to the bed as possible in an effort to minimize external load moment arms at the shoulder and back, to count to three in preparation for each trial, and to shift their body weight between their feet on each count.

Each trial commenced when the participant moved toward the patient after a starting signal. Participants had 15 seconds to complete the Turn Away, Turn Toward, and Reposition tasks and 25 seconds for the Lie-to-Sit and Sit-to-Chair trials. Trials lengths were established through pilot testing to provide sufficient time to perform each transfer at a typical self-selected pace. Once three repetitions of each task were completed, hand force estimations immediately followed. Participants verbally reported how hard they were working as a percentage of their maximum, in addition to verbally reporting how their exertion was distributed between their hands (e.g. $70 \%$ left vs $30 \%$ right). They then used the hand force dynamometers against a secured bar to replicate estimated hand forces, with hand force direction relative to the hand, task performance height and orientation relative to the participant takin into consideration.

\subsection{Data analysis}

Kinetic analysis consisted of data filtering, marker reconstruction, local joint coordinate system construction, and external joint moment calculations. Static calibration trials were completed prior to experimental trials, allowing reconstruction in subsequent frames. All raw kinematic data were low-pass filtered with a frequency of $6 \mathrm{~Hz}$ [26], and segment lengths and orthogonal coordinate systems were completed using ISB standards [27]. Locations of joint centres, along with participant weight, stature and estimated hand forces were used as inputs in a top-down inverse quasi-static model (similar to that of Dickerson et al. [28]) to determine time series moment data of the left and right shoulder as well as the low back. The recorded force from the hand force estimations was used throughout the trials. In order to prevent non-task movements from being considered, each trial was visually examined, and analysis was only completed on relevant frames. Peak resultant (vector norm) moments from each shoulder and the low back were then extracted for analysis.

Michigan's 3D static strength prediction program (3DSSPP, University of Michigan, Ann Arbor, MI, USA) was used to compare median $\left(50^{\text {th }}\right.$ percentile) population strength requirements to the untrained 
and trained condition demands. Video analysis of the self-selected techniques revealed that many participants selected similar styles for each task. Two styles were chosen for self-selected Lie-to-Sit, Sitto-Chair and Reposition postures, while one postural style was chosen for Turn Away and Turn Toward. The two styles for Lie-to-Sit and Reposition involved a push or a pull technique, while the Sit-to-Chair technique was completed at two different bed heights. The posture in which peak force application occurred was based on these techniques, and this posture was modeled in 3DSSPP, as well as the five recommended techniques [29]. Average height and weight of the 20 participants was used as anthropometric input for all techniques modeled in 3DSSPP. Analysis of these positions consisted of dividing the given required moment by the population mean strengths for both the untrained techniques and trained techniques to generate a required strength fraction.

\subsection{Statistical analysis}

All statistical analyses were performed using JMP 8.0 software (SAS Institute, North Carolina, USA). Statistical significance was set at $\alpha=0.05$ for all scenarios, with Student's T-Test post-hoc testing where appropriate. One way ANOVAs were completed separately for each of the five tasks using peak moment for the right shoulder, left shoulder and low back as dependent variables, with training state as the independent factor. The magnitude of change between pre- and post-training state on these peak moments was also expressed as a percentage change.

\section{Results}

Training had differential responses on shoulder and low back loading and strength requirements, and typically resulted in decreased low back loading and variable responses at the shoulder. Required strength fractions generally decreased at the low back, but was variable at the shoulder (Table 1). The results are divided by task in the following sections.

\subsection{Lie-to-Sit}

Training decreased peak moments in the low back and right shoulder, but not the left shoulder. The peak resultant low back moment decreased by $42 \%(p<0.01)$ (Table 2$)$. The left and right shoulders decreased by $26 \%(p<0.01)$ and $1 \%$, respectively $(p=0.96)$. Mean population strength fractions were decreased with technique changes following training in the left shoulder, right shoulder and low back by $36 \%, 42 \%$, and $33 \%$, respectively. However, the post-training technique increased right shoulder population strength fractions by $101 \%$, decreased the left shoulder by $38 \%$, and increased the low back by $12 \%$ compared to the untrained push technique.

\subsection{Reposition}

Training decreased low back, but not shoulder peak moments. Low back peak moments decreased by $43 \%(p<0.01)$, while the shoulders had non-significant results. Trained trials in the reposition task increased right shoulder peak moment loading by $15 \%(p=0.26)$, while decreasing the left shoulder by $19 \%(p=0.10)$. For mean population strength fractions, trained techniques compared to untrained pulls exertions had increased right shoulder and low back strength fractions, while decreasing or maintaining left shoulder strength fractions (Table 1). 
Table 1

Comparisons of mean population strength required for self-selected MPH techniques versus recommended MPH techniques. Percentages in Untrained and Trained rows represent percentage of mean population strength

\begin{tabular}{|c|c|c|c|c|}
\hline TASK & Condition & Right shoulder & Left shoulder & Low back \\
\hline \multirow[t]{5}{*}{ Lie-to-Sit } & Untrained (Pull) & $215 \%$ & $171 \%$ & $199 \%$ \\
\hline & Untrained (Push) & $68 \%$ & $160 \%$ & $118 \%$ \\
\hline & Trained & $136 \%$ & $99 \%$ & $132 \%$ \\
\hline & $\%$ Change (Pull) & $-36 \%$ & $-42 \%$ & $-33 \%$ \\
\hline & $\%$ Change (Push) & $+101 \%$ & $-38 \%$ & $+12 \%$ \\
\hline \multirow[t]{5}{*}{ Reposition } & Untrained (High Bed) & $151 \%$ & $157 \%$ & $175 \%$ \\
\hline & Untrained (Low Bed) & $140 \%$ & $179 \%$ & $149 \%$ \\
\hline & Trained & $169 \%$ & $157 \%$ & $182 \%$ \\
\hline & $\%$ Change (High Bed) & $+12 \%$ & $0 \%$ & $+4 \%$ \\
\hline & $\%$ Change (Low Bed) & $+20 \%$ & $-13 \%$ & $+3 \%$ \\
\hline \multirow[t]{5}{*}{ Sit-to-Chair } & Untrained (Pull) & $58 \%$ & $45 \%$ & $53 \%$ \\
\hline & Untrained (Push) & $70 \%$ & $89 \%$ & $50 \%$ \\
\hline & Trained & $109 \%$ & $88 \%$ & $56 \%$ \\
\hline & $\%$ Change (Pull) & $+89 \%$ & $+94 \%$ & $+5 \%$ \\
\hline & $\%$ Change (Push) & $+56 \%$ & $-1 \%$ & $+11 \%$ \\
\hline \multirow[t]{3}{*}{ Turn Away } & Untrained & $119 \%$ & $140 \%$ & $68 \%$ \\
\hline & Trained & $101 \%$ & $88 \%$ & $32 \%$ \\
\hline & $\%$ Change & $-15 \%$ & $-38 \%$ & $-53 \%$ \\
\hline \multirow[t]{3}{*}{ Turn toward } & Untrained & $70 \%$ & $85 \%$ & $73 \%$ \\
\hline & Trained & $94 \%$ & $102 \%$ & $35 \%$ \\
\hline & $\%$ Change & $+35 \%$ & $+19 \%$ & $-52 \%$ \\
\hline
\end{tabular}

Table 2

Peak joint moments (in Nm) during self-selected and trained MPH techniques. Significant differences between untrained and trained conditions within regions are bold

\begin{tabular}{llccc}
\hline TASK & Condition & Right shoulder & Left shoulder & Low back \\
\hline Lie-to-Sit & Untrained & 47.2 & $\mathbf{6 1 . 3}$ & $\mathbf{2 3 1 . 8}$ \\
& Trained & 46.9 & $\mathbf{4 5 . 3}$ & $\mathbf{1 3 3 . 5}$ \\
Reposition & Untrained & 50.6 & 62.8 & $\mathbf{1 9 5 . 1}$ \\
& Trained & 58.3 & 50.6 & $\mathbf{1 0 9 . 5}$ \\
Sit-to-Chair & Untrained & $\mathbf{2 7 . 3}$ & $\mathbf{2 9 . 3}$ & $\mathbf{1 9 7 . 0}$ \\
& Trained & $\mathbf{5 6 . 7}$ & $\mathbf{5 7 . 7}$ & $\mathbf{1 5 3 . 5}$ \\
Turn away & Untrained & 41.7 & 41.2 & $\mathbf{2 0 6 . 8}$ \\
& Trained & 35.7 & 36.6 & $\mathbf{1 1 4 . 7}$ \\
Toward & Untrained & 49.6 & $\mathbf{6 5 . 1}$ & $\mathbf{1 4 5 . 7}$ \\
& Trained & 46.1 & $\mathbf{5 3 . 0}$ & $\mathbf{1 1 2 . 0}$ \\
\hline
\end{tabular}

\subsection{Sit-to-Chair}

Training generated increases in peak moments in both shoulders, but decreases at the low back. Peak moments in the left shoulder and right shoulder increased during trained exertions by $97 \%$ and $107 \%$ respectively $(p<0.01$. The low back peak moment decreased by $45 \mathrm{Nm}(p=0.03)$. The recommended technique compared to the untrained techniques increased right shoulder strength fractions and had variable effects on the left shoulder and low back (Table 1).

\subsection{Turn away}

Low back peak moments decreased following training, but no changes existed in the shoulders. The low back moment decreased by $92 \mathrm{Nm}(p<0.01)$, a $44 \%$ decrease. Moments in the left and right 


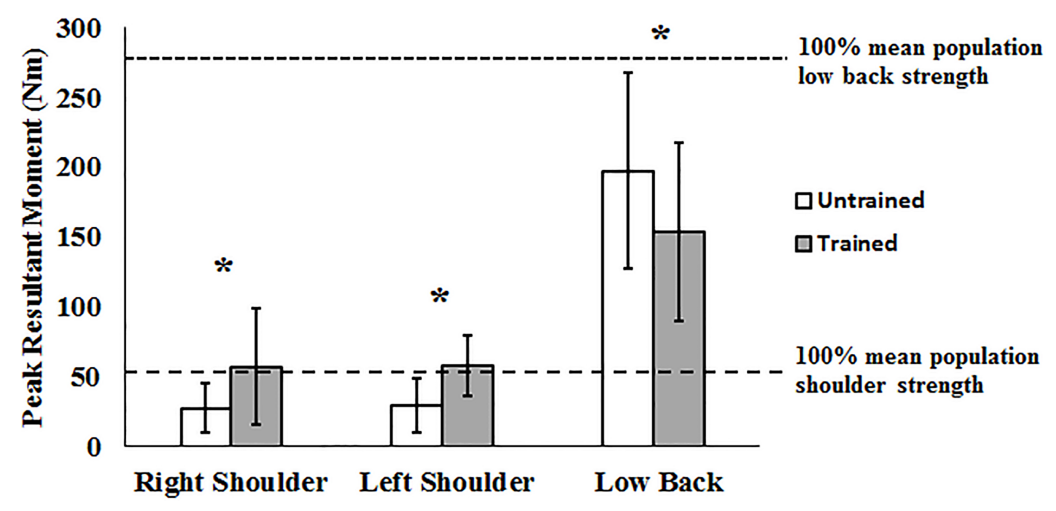

Fig. 2. Joint specific peak resultant moments for the Sit-to-Chair task when considering training state as an independent factor. Significant differences between pre- and post-training are marked by an asterisk.

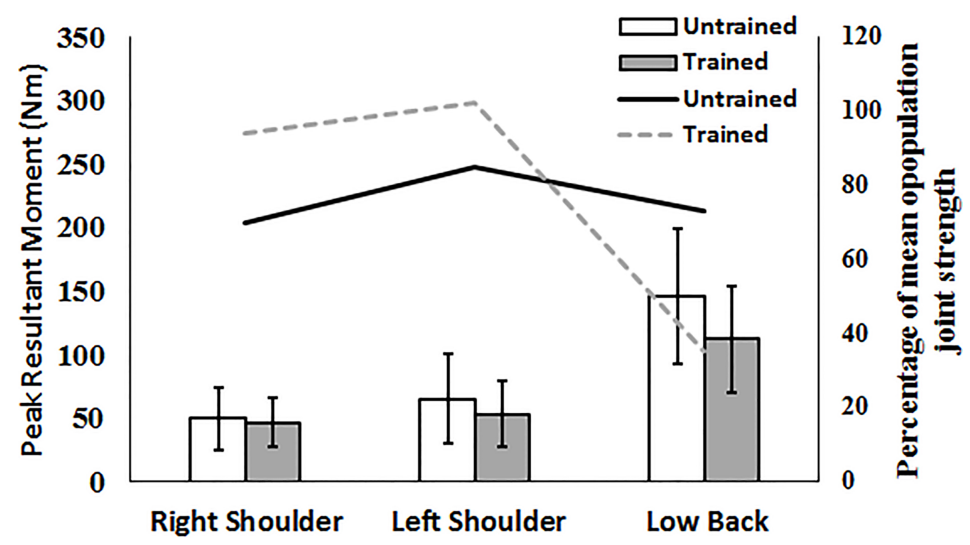

Fig. 3. Joint specific peak resultant moments and percentage of mean population strength for the Turn Toward task. Significant differences between joint specific peak resultant moments between pre- and post-training are marked by an asterisk. Columns are for peak resultant moments (PM in legend), lines are for percentage of mean population joint strength (JS in legend).

shoulders decreased by $11 \%$ and $14 \%$ respectively, but these were not statistically significant $(p=$ 0.09 and 0.13 , respectively). Training decreased mean population strength fractions in the left and right shoulders and the low back by $15 \%, 38 \%$ and $53 \%$, respectively.

\subsection{Turn toward}

Decreases existed in the left shoulder and the low back, but not the right shoulder. The left shoulder moment decreased by $12.1 \mathrm{Nm}$ or $18 \%(p=0.03)$, while the low back decreased by $33 \mathrm{Nm}(p<0.01)$, a $23 \%$ change. The right shoulder decreased insignificantly by $7 \%(p=0.43)$. Trained exertions increased mean population strength fractions required in the shoulders (35\% for the right, 19\% for the left), but decreased the low back by $52 \%$.

\section{Discussion}

The focus of this research was to examine if techniques designed to decrease low back demands transferred these exposures to the shoulders, or if overall loading decreased holistically. Peak resultant 
magnitudes were reduced for the low back for every task, and reductions occurred for the shoulders in some tasks, but demands increased after training for others, indicating a spectrum of exposures. Peak moments at the low back decreased by $22-44 \%$, depending on the task. This confirms previous reports of decreased low back exposures with recommended manual patient handling techniques $[9,24,30]$. Peak resultant moments at the right shoulder increased using the recommended techniques for two of the five tasks, with no change in a third task and increases in two tasks; left shoulder peak moments decreased in four of five scenarios. The identified moment increases suggest possible exposure transfers from the low back to the shoulder.

These changes were then converted into a common scale by replicating the postures assumed by the participants during peak force exertion were within a static strength analysis program (3DSSPP) and interpreted. This provided an estimate of how capable the study population is of performing the techniques compared to population normative strength data [29]. For several tasks, the required strength at the shoulders and low back exceeded the mean population strength while using the recommended techniques, increasing overexertion risk [31,32]. In many cases, using the recommended techniques increased the percentage of mean population strength required to complete the tasks at the shoulders. The recommended techniques required at least $88 \%$ of the mean population strength at both shoulders for all tasks, and every scenario except Turn Away resulted in increased strength requirements compared to novice techniques in at least one shoulder.

Joint demand loading changes are readily identified in the Turn Toward and Sit-to-Chair tasks. In the Turn Toward task, the untrained technique balanced exposures nearly equally amongst all three joints, with each joint requiring approximately $75 \%$ of the mean population joint strength. Once the recommended technique was adopted, the low back mean population strength fraction decreased to $35 \%$, while the left and right shoulders increased to nearly $100 \%$ mean joint strength fraction. This confirms previous recognition of the Turn Toward task as a stressful nursing task [33]. The largest increase occurred in the Sit-to-Chair task, with peak resultant moments at the right and left shoulders increasing by $107 \%$ and $97 \%$, respectively, while the low back moment decreased by $22 \%$. This task is frequently targeted for replacement with mechanical lift assists [34]. The strength requirement changes establish both the overall high demand of these MPH tasks while highlighting the potential for transfer of demand between joints for certain tasks.

Field examination of manual patient handling is challenging due to complex interaction between nurse and patient in various situations, and this generated limitations in our work. At present, there is no benchmark for recording separate hand forces in manual patient handling scenarios [35,36]. The current research did not want to interfere with participant techniques or generate compensations through encumbrance of heavy or awkward equipment, and hand demands (such as holding dynamometers during transfers) are known to affect strategies [37,38]. Absolute direction of the force applied was estimated, and the use of this method limits analysis to calculation of peak moments, potentially over- or underestimating peak joint moments. However, this study was primarily interested in changes related to training and task type and relative rather than absolute magnitudes. The estimated magnitudes were of similar shoulder moment magnitudes for previous reposition tasks, confirming the reasonableness of the approach [19]. Finally, the set of manual patient handling tasks was not exhaustive, and each participant was limited to approaching the left side of the bed. Approaching from the opposite side of the bed may have generated bilaterally opposite exposures, but anecdotal evidence from practicing nurses suggests that handedness is not typically considered in manual patient handling. 


\section{Conclusion}

This study supports a recommended of continued implementation of several of the manual patient handling techniques; however, certain cautions should be considered for some transfers. The recommended techniques were aimed specifically at lowering low back injury exposures, but may transferring demands to the shoulders. The Turn Toward and Sit-to-Chair tasks appear to adversely affect the shoulder the most, suggesting they should be avoided if possible. Future investigations may benefit from developing novel holistic patient handling techniques that do not transfer demands to other areas of the body.

\section{Conflict of interest}

The authors have no conflict of interest to report.

\section{References}

[1] Garg A, Owen B, Beller D, Banaag J. A biomechanical and ergonomic evaluation of patient transferring tasks: bed to wheelchair and wheelchair to bed. Ergonomics. 1991; 34(3): 289-312.

[2] Owen B, Keene K, Olson S. An ergonomic approach to reducing back/shoulder stress in hospital nursing personnel: a five year follow up. Int J Nurs Stud. 2002; 39(3): 295-302.

[3] Nelson A, Baptiste A. Evidence-based practices for safe patient handling and movement. Orthop Nurs. 2006; 25(6): 366-79.

[4] WSIB. 2015 Statistical Report, 2015.

[5] Bureau of Labor Services. Workplace injuries and illnesses in 2007, 2008.

[6] Garg A, Owen B. Prevention of back injuries in healthcare workers. Int J Ind Ergon. 1994; 14(4): 315-31.

[7] Daynard D, Yassi A, Cooper J, Tate R, Norman R, Wells R. Biomechanical analysis of peak and cumulative spinal loads during simulated patient-handling activities: a substudy of a randomized controlled trial to prevent lift and transfer injury of health care workers. Appl Ergon. 2001; 32(3): 199-214.

[8] Keir P, MacDonell C. Muscle activity during patient transfers: a preliminary study on the influence of lift assists and experience. Ergonomics. 2004; 47(3): 296-306.

[9] Schibye B, Hansen A, Hye-Knudsen C, Essendrop M, Böcher M, Skotte J. Biomechanical analysis of the effect of changing patient-handling technique. Appl Ergon. 2003; 34(2): 115-23.

[10] Fujishiro K, Weaver J, Heaney C, Hamerick C, Marras W. The effect of ergonomic interventions in healthcare facilities on musculoskeletal disorders. Am J Ind Med. 2005; 48: 338-47.

[11] Garg A, Owen B, Carlson B. An ergonomic evaluation of nursing assistants' job in a nursing home. Ergonomics. 1992; 35(9): 979-95.

[12] Engst C, Chhokar R, Miller A, Tate R, Yassi A. Effectiveness of overhead lifting devices in reducing the risk of injury to care staff in extended care facilities. Ergonomics. 2005; 48(2): 187-99.

[13] Evanoff B, Wolf L, Aton E, Canos J, Collins J. Reduction in injury rates in nursing personnel through introduction of mechanical lifts in the workplace. Am J Ind Med. 2003; 44(5): 451-7.

[14] Vendittelli D, Penprase B, Pittiglio L. Musculoskeletal Injury Prevention for New Nurses. Workplace Health Saf [Internet]. 2016; 64(12): 573-85.

[15] Kneafsey R, Haigh C. Learning safe patient handling skills: Student nurse experiences of university and practice based education. Nurse Educ Today. 2007; 27: 832-9.

[16] Kneafsey R. Developing skills in safe patient handling: Mentors' views about their role in supporting student nurses. Nurse Educ Pract. 2010; 7: 365-72.

[17] Cornish J, Jones A. Factors affecting compliance with moving and handling policy: Student nurses “ views and experiences. Nurse Educ Pract. 2007; 10: 96-100.

[18] Cornish J, Jones A. Evaluation of moving and handling training for pre-registration nurses and its application to practice. Nurse Educ Pract. 2010; 7: 128-34.

[19] Yassi A. Development of a method for quantifying biomechanical risk factors associated with manual and mechanically assisted patient handling. Richmond, BC, Canada: Worksafe BC, 2005. 
[20] Gagnon M, Smyth G. Muscular mechanical energy expenditure as a process for detecting potential risks in manual materials handling. J Biomech. 1991; 24(3-4): 191-203.

[21] Menzel N, Brooks S, Bernard T, Nelson A. The physical workload of nursing personnel: association with musculoskeletal discomfort. Int J Nurs Stud. 2004; 41(8): 859-67.

[22] Kingma I, Looze de M. Validation of a full body 3-D dynamic linked segment model. Hum Mov Sci [Internet]. 1996; [cited 2014 Sep 24]; 833-60.

[23] Marshall M, Armstrong T, Ebersole M. Verbal estimation of peak exertion intensity. Hum Factors. 2004; 46(7): 697-710.

[24] Skotte J, Essendrop M, Hansen A, Schibye B. A dynamic 3D biomechanical evaluation of the load on the low back during different patient-handling tasks. J Biomech. 2002; 35(10): 1357-66.

[25] Gordon C, Churchill T, Clauser C, Bradtmiller B, McConville J, Tebbets I, et al. 1988 Anthropometric Survey of U.S. Army Personnel: Methods and Summary Statistics. Tech Rep NATICK/TR-89/044 Natick, MA; US Army Natick Res Dev Eng Cent (AD A225 094). 1989.

[26] Winter D. Biomechanics and Motor Control of Human Movement, 4th Edition, 2009.

[27] Wu G, van Der Halm F, Veeger H, Maksous M, Van Roy P, Anglin C, et al. ISB recommendation on definitions for the reporting of human joint motion - Part II: shoulder, elbow, wrist and hand. J Biomech. 2005; 38: 981-92.

[28] Dickerson C, Martin B, Chaffin D. The relationship between shoulder torques and the perception of muscular effort in loaded reaches. Ergonomics. 2006; 49(11): 1036-51.

[29] Chaffin D. Development of computerized human static strength simulation model for job design. Hum Factors Ergon Manuf. 1997; 7(4): 305-22.

[30] Skotte J, Fallentin N. Low back injury risk during repositioning of patients in bed: the influence of handling technique, patient weight and disability. Ergonomics. 2008; 51(7): 1042-52.

[31] Chaffin D, Herrin G, Keyserling W. Preemployment strength testing: an updated position. J Occup Med. 1978; 20(6): 403-8.

[32] Keyserling W, Herring G, Chaffin D. Isometric strength testing as means of controlling medical incidents on strenuous jobs. J Occup Med. 1980; 22(5): 332-6.

[33] Zhuang Z, Stobbe T, Collins J, Hongwei H, Hobbs G. Psychophysical assessment of assistive devices for transferring patients/residents. Appl Ergon. 1999; 31(1): 35-44.

[34] Santaguida P, Pierrynowski M, Goldsmith C, Fernie G. Comparison of cumulative low back loads of caregivers when transferring patients using overhead and floor mechanical lifting devices. Clin Biomech. 2005; 20(9): 906-16.

[35] Radwin R, Masters G, Lupton F. A linear force-summing hand dynamometer independent of point of application. Appl Ergon. 1991; 22(5): 339-45.

[36] Bao S, Silverstein B. Estimation of hand force in ergonomic job evaluations. Ergonomics, 48(3): 288-301. Ergonomics. 2005; 48(3): 288-301.

[37] Antony N, Keir P. Effects of posture, movement and hand load on shoulder muscle activity. J Electromyogr Kinesiol. 2010; 20(2): 191-8.

[38] Brookham R, Wong J, Dickerson C. Upper limb posture and submaximal hand tasks influence shoulder muscle activity. Int J Ind Ergon. 2010; 40(3): 337-44. 